\title{
On the Structure of the Lambda 1405
}

\author{
Jonathan M. M. Hall, Waseem Kamleh
}

Special Research Centre for the Subatomic Structure of Matter (CSSM),

School of Chemistry and Physics, University of Adelaide, South Australia 5005, Australia

Derek B. Leinweber*it

Special Research Centre for the Subatomic Structure of Matter (CSSM),

School of Chemistry and Physics, University of Adelaide, South Australia 5005, Australia

E-mail: derek. leinweber@adelaide.edu.au

\section{Benjamin J. Menadue}

Special Research Centre for the Subatomic Structure of Matter (CSSM),

School of Chemistry and Physics, University of Adelaide, South Australia 5005, Australia

National Computational Infrastructure (NCI),

Australian National University, Australian Capital Territory 0200, Australia

\section{Benjamin J. Owen}

Special Research Centre for the Subatomic Structure of Matter (CSSM),

School of Chemistry and Physics, University of Adelaide, South Australia 5005, Australia

\section{Anthony W. Thomas, Ross D. Young}

Special Research Centre for the Subatomic Structure of Matter (CSSM) and

ARC Centre of Excellence for Particle Physics at the Terascale (CoEPP),

School of Chemistry and Physics, University of Adelaide, South Australia 5005, Australia

For almost 50 years the structure of the $\Lambda(1405)$ resonance has been a mystery. Recently, a new lattice QCD simulation showing that its strange magnetic form factor vanishes, together with a comprehensive Hamiltonian analysis of the lattice QCD energy levels, has unambiguously established that the structure is dominated by a bound anti-kaon-nucleon component [1]. Here we present supplementary information for Ref. [1] including a presentation of the relevant Hamiltonian effective field theory and an illustration of the volume dependence of the results and their connection to the infinite volume limit of Nature.

The 32nd International Symposium on Lattice Field Theory

23-28 June, 2014

Columbia University New York, NY

\footnotetext{
*Speaker.

†e thank PACS-CS Collaboration for making their $2+1$ flavor configurations available and acknowledge the ongoing support of the ILDG. This research was undertaken with the assistance of resources at the NCI National Facility in Canberra, Australia, and the iVEC facilities at Murdoch University (iVEC@Murdoch) and the University of Western Australia (iVEC@UWA). These resources are provided through the National Computational Merit Allocation Scheme and the University of Adelaide Partner Share supported by the Australian Government. We also acknowledge eResearch SA for their support of our supercomputers. This research is supported by the Australian Research Council through the ARC Centre of Excellence for Particle Physics at the Terascale, and through Grants DP120104627 (DBL), DP140103067 (DBL and RDY), FT120100821 (RDY) and FL0992247 (AWT).
} 


\section{Introduction}

For almost 50 years the structure of the $\Lambda(1405)$ resonance has been a mystery. Even though it contains a relatively massive strange quark and has odd parity, both of which should increase its mass, it is, in fact, lighter than any other excited spin-1/2 baryon. Identifying the explanation for this observation has challenged theorists since its discovery in the 1960s [2]. While the quantum numbers of the $\Lambda(1405)$ can be described by three quarks, (uds), its totally unexpected position in the spectrum has rendered its structure quite mysterious.

Recently, the very first Lattice QCD calculation of the electromagnetic form factors of the $\Lambda(1405)$ were presented [1]. This calculation reveals the vanishing of the strange quark contribution to the magnetic form factor of the $\Lambda(1405)$ in the regime where the masses of the up and down quarks approach their physical values.

This result is very naturally explained if the state becomes a molecular $\bar{K} N$ bound state in that limit. In this case the strange quark is confined within a spin-0 kaon and has no preferred spin orientation. Because the anti-kaon has zero orbital angular momentum in order to conserve parity, the strange quark cannot contribute to the magnetic form factor of the $\Lambda(1405)$. On the other hand, if the $\Lambda(1405)$ were a $\pi \Sigma$ state or an elementary three-quark state the strange quark must make a sizable contribution to the magnetic form factor. Only if the $\bar{K} N$ component in the structure of the $\Lambda(1405)$ is dominant would one expect to find a vanishing strange-quark magnetic form factor.

Upon combining the observation of a vanishing strange quark magnetic form factor with a Hamiltonian effective-field-theory analysis of the structure of the state as a function of its light quark mass, which shows $\bar{K} N$ dominance and a rapidly decreasing wave function renormalisation constant in the same limit, one concludes that this long-standing problem has been unambiguously resolved [1].

In this presentation we provide additional information in support of Ref. [1] including a presentation of the relevant Hamiltonian effective field theory and an illustration of the volume dependence of the results and their connection to the infinite volume limit of Nature.

\section{Hamiltonian Model}

In this section we present the construction and analysis of the finite-volume Hamiltonian effective field theory. We follow a procedure similar to that for the $\Delta \rightarrow N \pi$ analysis [3]. However, in the present case multiple meson-baryon channels are considered. In constructing the Hamiltonian, the four octet meson-baryon interaction channels of the $\Lambda(1405)$ are included: $\pi \Sigma, \bar{K} N, K \Xi, \eta \Lambda$.

We begin by writing the Hamiltonian $H$ as the sum of free and interacting Hamiltonians, $H=H_{0}+H_{I}$. The rows and columns of $H$ represent the magnitudes of the three-momenta available to the meson-baryon intermediate states dressing the bare $\Lambda(1405)$ state. As we work with total momentum zero, the meson and the baryon will each carry the same magnitude of momentum in a back-to-back orientation. In a finite periodic volume, momentum is quantised. Working on a cubic volume of extent $L$ on each side, it is convenient to define the momentum magnitudes

$$
k_{n}=\sqrt{n_{x}^{2}+n_{y}^{2}+n_{z}^{2}} \frac{2 \pi}{L},
$$


with $n_{i}=0,1,2, \ldots$ and integer $n=n_{x}^{2}+n_{y}^{2}+n_{z}^{2}$. As there are permutations of the $n_{i}$ that give rise to the same momentum magnitude, we also introduce the integer $C_{3}(n)$ as a combinatorial factor equal to the number of unique permutations of $\pm n_{x}, \pm n_{y}$ and $\pm n_{z}$. For example, the lowest lying nontrivial momentum available on the lattice, where one direction has the magnitude of $2 \pi / L$, has $C_{3}(1)=6$. The result recognises three positions for the non-trivial momentum and a factor of two associated with whether the meson or the baryon carries the positive momentum.

The non-interacting Hamiltonian $H_{0}$ has diagonal entries corresponding to the relativistic noninteracting meson-baryon energies available on the finite periodic volume at total three-momentum zero. It also includes a single-particle state with bare mass, $m_{0}+\alpha_{0} m_{\pi}^{2}$. The parameters $m_{0}$ and $\alpha_{0}$ are to be constrained by the lattice QCD results. Denoting each meson-baryon energy by $\omega_{M B}\left(k_{n}\right)=\omega_{M}\left(k_{n}\right)+\omega_{B}\left(k_{n}\right)$, with $\omega_{A}\left(k_{n}\right) \equiv \sqrt{k_{n}^{2}+m_{A}^{2}}$, the non-interacting Hamiltonian takes the form

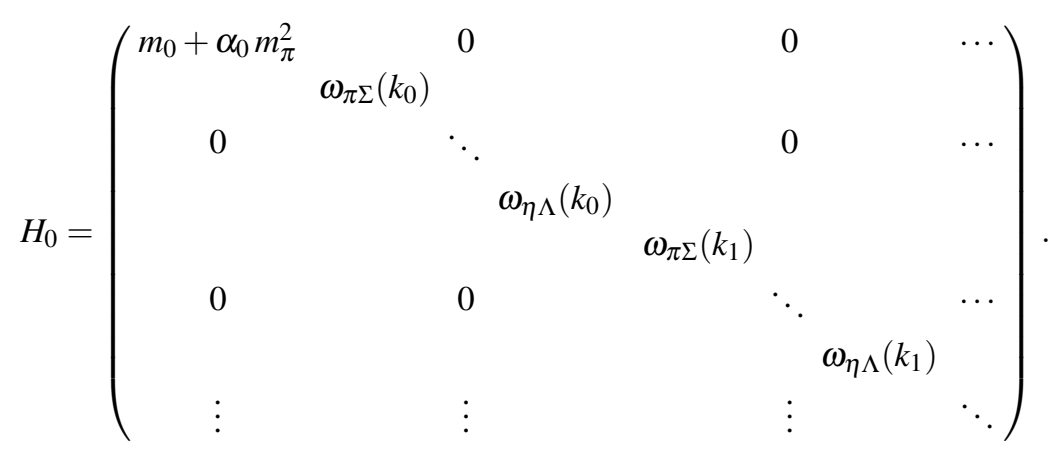

In the present model the interaction entries describe the coupling of the single-particle state to the two-particle meson-baryon states.

$$
H_{I}=\left(\begin{array}{cccccc}
0 & g_{\pi \Sigma}\left(k_{0}\right) & \cdots & g_{\eta \Lambda}\left(k_{0}\right) g_{\pi \Sigma}\left(k_{1}\right) \cdots g_{\eta \Lambda}\left(k_{1}\right) \cdots \\
g_{\pi \Sigma}\left(k_{0}\right) & 0 & \cdots & & & \\
\vdots & \vdots & 0 & & & \\
g_{\eta \Lambda}\left(k_{0}\right) & & & \ddots & & \\
g_{\pi \Sigma}\left(k_{1}\right) & & & & & \\
\vdots & & & & &
\end{array}\right) .
$$

Each entry represents the $S$-wave interaction energy of the $\Lambda(1405)$ with one of the four channels at a certain value for $k_{n}$. The form of the interaction is derived from effective field theory, and includes the relevant finite-volume factors [3]

$$
g_{M B}\left(k_{n}\right)=\left(\frac{\kappa_{M B}}{16 \pi^{2} f_{\pi}^{2}} \frac{C_{3}(n)}{4 \pi}\left(\frac{2 \pi}{L}\right)^{3} \omega_{M}\left(k_{n}\right) u^{2}\left(k_{n}\right)\right)^{1 / 2} .
$$

$f_{\pi}=92.4 \mathrm{MeV}$ represents the pion decay constant and $u^{2}\left(k_{n}\right)$ is a regulator function. For the purposes of this model, $u\left(k_{n}\right)$ takes the form of a dipole regulator, with a regularization scale of $\Lambda=0.8 \mathrm{GeV}$. It has been shown in previous investigations that the regulator dependence is small in the extraction of resonance parameters near the physical pion mass [4]. The coupling $\kappa_{M B}$ is 

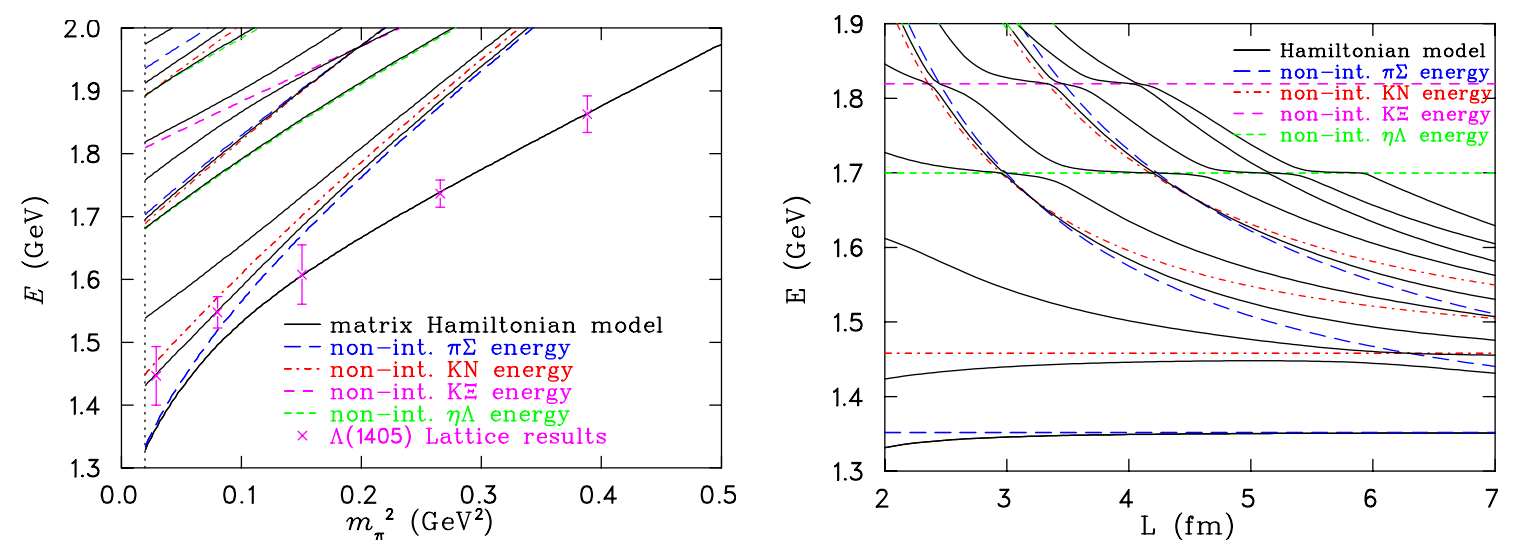

Figure 1: (left) The quark-mass dependence $\left(m_{q} \propto m_{\pi}^{2}\right)$ of the lowest-lying $\Lambda(1405)$ states observed in our lattice QCD calculations [1] is illustrated by the discrete points at each of the pion masses available in the PACS-CS ensembles. The low-lying energy spectrum of our Hamiltonian model (solid curves) constrained to the Lattice QCD results (discrete points) is also illustrated. The associated non-interacting meson-baryon basis states are illustrated by the dashed curves. (right) The volume dependence of the spin-1/2 odd-parity $\Lambda$ spectrum obtained from our Hamiltonian effective field theory analysis of our lattice results at $m_{\pi}=156$ MeV. $L$ denotes the length of the $L^{3}$ volume.

related to the $S U(3)$-flavour singlet couplings of the octet mesons and baryons via [5, 6]

$$
\kappa_{\pi \Sigma}=3 \xi_{0}, \quad \kappa_{\bar{K} N}=2 \xi_{0}, \quad \kappa_{K \Xi}=2 \xi_{0}, \quad \kappa_{\eta \Lambda}=\xi_{0},
$$

with $\xi_{0}=0.75$, chosen in order to ensure that the $\pi \Sigma$ decay width of the $\Lambda(1405)$ takes the physical value of $50 \pm 2 \mathrm{MeV}$ [7] at the physical pion mass, in the infinite-volume limit.

The eigenvalue equation corresponding to the Hamiltonian model presented here takes the following simple form which is consistent with chiral effective field theory

$$
\lambda=m_{0}+\alpha_{0} m_{\pi}^{2}-\sum_{M, B} \sum_{n=0}^{\infty} \frac{g_{M B}^{2}\left(k_{n}\right)}{\omega_{M B}\left(k_{n}\right)-\lambda} .
$$

$M$ and $B$ denote the intermediate meson-baryon pairs, with coupling $g_{M B}\left(k_{n}\right)$ provided in Eq. (2.4). $\lambda$ is the energy eigenvalue of the $S$-wave interaction, which occurs on both sides of the equation. As $\lambda$ is finite, the pole in the denominator of the right-hand side is never accessed. A nontrivial mixing of states occurs and the bare mass $m_{0}+\alpha_{0} m_{\pi}^{2}$ encounters self-energy corrections that lead to avoided level-crossings in the finite-volume energy eigenstates.

To solve this Hamiltonian system, the dgeev routine from the LAPACK software library is used to obtain the eigenvalues and eigenvectors of $H$. The energy eigenvalues of the matrix may be fitted to their corresponding lattice QCD values by minimising the chi-square function for the parameters $m_{0}$ and $\alpha_{0}$ at different values of $m_{\pi}^{2}$. The low-lying energy eigenvalues fit to the lattice QCD results are illustrated in Fig. 1. The scale is set via the Sommer parameter with $r_{0}=0.492$ fm [8].

\section{Discussion}

The three heaviest quark masses considered on the lattice correspond to a stable odd-parity $\Lambda(1405)$, as the $\pi \Sigma$ threshold energy exceeds that of the $\Lambda(1405)$. However, as the physical pion 
mass is approached, the $\pi \Sigma$ threshold energy decreases and a nontrivial mixing of states associated with an avoided level crossing of the transitioning $\pi \Sigma$ threshold occurs. At the lightest two quark masses considered, the $\Lambda(1405)$ corresponds to the second state of the Hamiltonian model with a $\pi \Sigma$-dominated eigenstate occupying the lowest energy position.

The right-hand panel of Fig. 1 presents the volume dependence of the odd-parity $\Lambda$ spectrum obtained from our Hamiltonian effective field theory analysis of our lattice results at the lightest quark mass providing $m_{\pi}=156 \mathrm{MeV}$. The second state, identified as the $\Lambda(1405)$ via the quark mass dependence of the spectrum presented in the left-hand panel of Fig. 1, undergoes an avoided level crossing just beyond $L=6 \mathrm{fm}$ to become the third state in the spectrum. Here the second state turns down to approach the $\pi \Sigma$ scattering state where each hadron carries one unit of lattice momenta, $2 \pi / L$. The process of avoided level crossings will repeat as more $\pi \Sigma$ scattering states have their energy drop below the $\Lambda(1405)$ energy as $L$ increases.

The correlation matrix approach $[9,10]$ was used to determine the superposition of interpolators required to isolate the $\Lambda(1405)$ on the lattice [11]. This discovery of a low-lying $\Lambda(1405)$ mass [11] has since been independently confirmed [12]. The success of our approach in accurately isolating the $\Lambda(1405)$, even at the lightest quark mass considered, is best demonstrated by the long Euclidean-time single-state stability of the $\Lambda(1405)$ two-point correlation function presented in Fig. 2 of Ref. [13]. We note this success is realised without resort to two-particle interpolators.

The results of Fig. 1 emphasize how the near-by $\bar{K} N$ threshold realised in the infinite-volume limit of Nature does not sit next to the $\Lambda(1405)$ in a finite volume with $L \sim 3 \mathrm{fm}$. Rather the mixing of states in the finite volume renders any infinite-volume concerns on the role of nearby thresholds irrelevant.

The vanishing of the strange quark contribution to the magnetic form factor of the state observed in our lattice calculation at the lightest quark mass signals the creation of a $\bar{K} N$ state from three-quark operators. The success of creating a five-quark state from a three quark operator is reminiscent of the dream of observing string breaking in the static quark potential. There the hope was that the sea-quark loops in the vacuum would become manifest in a flattening of the potential at large separations. However the dream was not realised and explicit quark degrees of freedom had to be introduced by hand in a correlation matrix analysis. In contrast, the sea-quark loops in the vacuum are manifest in the magnetic form factor of the $\Lambda(1405)$, without resort to explicit five-quark operators.

\section{Infinite-Volume Limit}

Having confirmed that the $\Lambda(1405)$ state observed on the lattice is best described as a molecular $\bar{K} N$ bound state, it remains to demonstrate the connection between the finite-volume lattice eigenstates and the infinite-volume resonance found in Nature. The quark-mass behaviour of the $\Lambda(1405)$ energy in the infinite-volume limit can be reconstructed from the finite-volume Hamiltonian model by considering the principal-value continuum versions of the loop integral contributions from all channels with the appropriate physical hadron masses. The resonance energy of the $\Lambda(1405)$ in infinite volume is

$$
E_{\Lambda 1405}=m_{0}^{\mathrm{fit}}+\alpha_{0}^{\mathrm{fit}} m_{\pi}^{2}+\sum_{M, B} \frac{\kappa_{M B}}{16 \pi^{2} f_{\pi}^{2}} \mathscr{P} \int_{0}^{\infty} \mathrm{d} k \frac{k^{2} \omega_{M}(k) u^{2}(k)}{E_{\Lambda 1405}-\omega_{M B}(k)},
$$




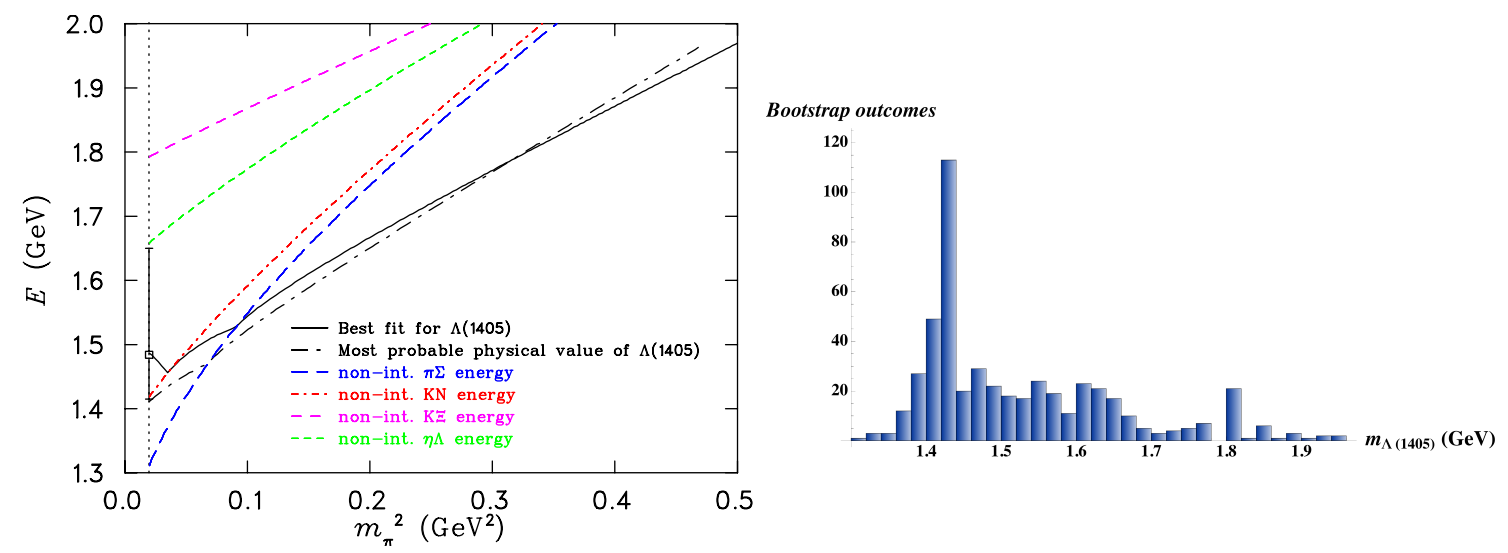

Figure 2: (left) The quark mass dependence of the infinite-volume reconstruction of the $\Lambda(1405)$ energy is illustrated as a function of the squared pion-mass, $m_{\pi}^{2}$. Both the fit to the central values of the lattice QCD results and the most probable mass dependence observed in the bootstrap ensemble analysis of the lattice results are illustrated. (right) The statistical distribution of the infinite-volume $\Lambda(1405)$ resonance energy at the physical pion mass obtained from a bootstrap analysis of the Lattice QCD results.

where $\mathscr{P}$ indicates that the principal value integral is performed. This integral represents the infinite-volume version of the loop sum appearing in Eq. (2.6). Since $E_{\Lambda 1405}$ appears on both sides of Eq. (4.1), it is best solved iteratively by scanning over a range of possible values of $E_{\Lambda 1405}$.

The result is shown in the left-hand panel of Fig. 2 as a solid black line. The dashed lines illustrate the non-interacting infinite-volume $S$-wave threshold energies, which each induce a nonanalytic cusp in the quark-mass dependence of the self energy.

To obtain an estimate of the statistical uncertainty in the $\Lambda(1405)$ energy, a bootstrap analysis is performed. This is achieved by repeating the minimisation of the chi-square to obtain fitted values of $m_{0}$ and $\alpha_{0}$ for separate bootstrap ensembles of lattice QCD data. The bootstraps are calculated by altering the value of each lattice data point by a Gaussian-distributed random number, weighted by the uncertainty at each point in $m_{\pi}^{2}$. The statistical distribution of values for the $\Lambda(1405)$ mass at the physical pion mass and infinite volume, for 500 bootstrap configurations, is shown in the form of a histogram, displayed in the right-hand panel of Fig. 2. The plot has an unconventional distribution due to cusps in the extrapolation associated with the opening of decay channel(s). The bootstrap error analysis provides a resonance energy of $1.48_{-0.07}^{+0.17} \mathrm{GeV}$.

The distribution of the bootstrap analysis is sharply peaked around the most probable value of $1.41 \mathrm{GeV}$ in good agreement with experiment. We illustrate the most probable outcome for the $\Lambda(1405)$ mass dependence by the dot-dash curve in the left-hand panel of Fig. 2 labeled "Most probable physical value of $\Lambda(1405)$." In this case only the $\pi \Sigma$ threshold induces a cusp and the ordering of the $\pi \Sigma$ threshold, the $\Lambda(1405)$ energy, and the $\bar{K} N$ threshold realised in Nature is reproduced.

\section{Wave Function Renormalisation}

The pion-mass dependence of the $\Lambda(1405)$ can be further examined via the wave function renormalisation, $Z_{2}$, defined as the ratio of the renormalised coupling constant to the bare coupling constant. It can be calculated using 


$$
Z_{2}=\left.\frac{1}{1-\frac{\partial}{\partial E} \Sigma^{\operatorname{loops}}\left(m_{\pi}^{2}, E\right)}\right|_{E=E_{\Lambda 1405}} .
$$

This quantity describes the extent to which the resonance behaves like an elementary particle where $Z_{2}=1$, or a bound state where $Z_{2}=0$.

Results for $Z_{2}$ are illustrated in Fig. 3. For large pion masses, the $\Lambda(1405)$ is more like an elementary particle. In addition, the finiteand infinite-volume values of $Z_{2}$ match very closely. This is to be expected in a quark-mass region that lies far from a resonance. As the pion mass becomes smaller, the Hamiltonian model suggests stronger overlap with neighbouring particle states associated with a molec-

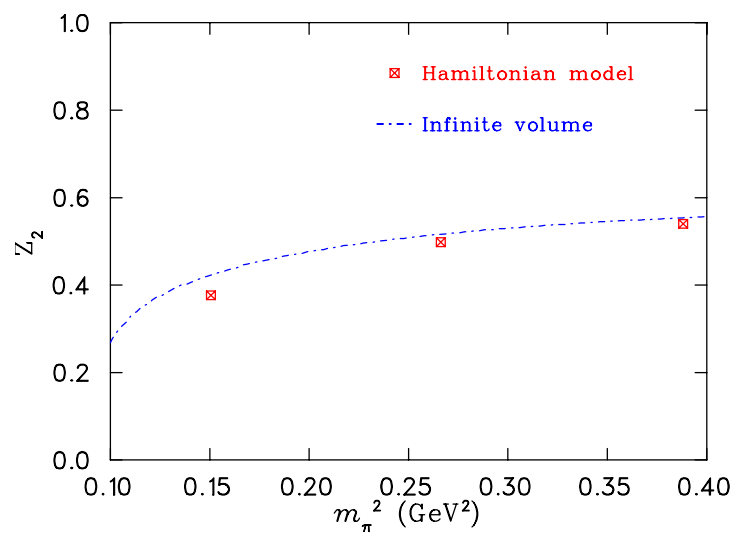

Figure 3: Wave function renormalisation factor $Z_{2}$ as a function of pion mass. While $Z_{2}=1$ corresponds to an elementary particle, $Z_{2}=0$ corresponds to a bound state. For $m_{\pi}<216 \mathrm{MeV}$, the infinite-volume $Z_{2}$ cannot be defined.

ular bound state. The probability of the 3-quark component decreases and the eigenstate is predominantly a $\bar{K} N$ bound state. For $m_{\pi}<216 \mathrm{MeV}$, the $\pi \Sigma$ channel opens, and the infinite-volume wave-function renormalisation can no longer be defined in the resonant region.

\section{References}

[1] J. M. M. Hall, W. Kamleh, D. B. Leinweber, B. J. Menadue, B. J. Owen, A. W. Thomas, and R. D. Young, arXiv:1411.3402.

[2] A. Engler, H. Fisk, R. Kraemer, C. Meltzer, and J. Westgard, Phys. Rev. Lett. 15 (1965) 224.

[3] J. Hall, A. C. P. Hsu, D. Leinweber, A. Thomas, and R. Young, Phys. Rev. D87 (2013) 094510, [arXiv:1303.4157].

[4] D. B. Leinweber, A. W. Thomas, and R. D. Young, Phys. Rev. Lett. 92 (2004) 242002, [hep-lat/0302020].

[5] E. Veit, B. K. Jennings, R. Barrett, and A. W. Thomas, Phys. Lett. B137 (1984) 415.

[6] E. Veit, B. K. Jennings, A. W. Thomas, and R. Barrett, Phys. Rev. D31 (1985) 1033.

[7] Particle Data Group Collaboration, J. Beringer et al., Phys. Rev. D86 (2012) 010001.

[8] PACS-CS Collaboration Collaboration, S. Aoki et al., Phys. Rev. D79 (2009) 034503, [arXiv:0807.1661].

[9] C. Michael, Nucl. Phys. B259 (1985) 58.

[10] M. Luscher and U. Wolff, Nucl. Phys. B339 (1990) 222-252.

[11] B. J. Menadue, W. Kamleh, D. B. Leinweber, and M. S. Mahbub, Phys. Rev. Lett. 108 (2012) 112001, [arXiv:1109.6716].

[12] G. P. Engel, C. Lang, and A. Schafer, Phys. Rev. D87 (2013), no. 3 034502, [arXiv: 1212 . 2032].

[13] B. J. Menadue, W. Kamleh, D. B. Leinweber, M. S. Mahbub, and B. J. Owen, PoS LATTICE2013 (2013) 280, [arXiv:1311.5026]. 